\title{
Electrical network reduction for load flow and short-circuit calculations using powerfactory software
}

\author{
Funso K. Ariyo \\ ${ }^{1}$ Department of Electronic and Electrical Engineering, Ile-Ife, Nigeria \\ ${ }^{2}$ Obafemi Awolowo University, Ile-Ife, Nigeria
}

Email address:

funsoariyo@yahoo.com (F. K. Ariyo)

\section{To cite this article:}

Funso K. Ariyo. Electrical Network Reduction for Load Flow and Short-Circuit Calculations Using Power Factory Software, American Journal of Electrical Power and Energy Systems. Vol. 2, No. 1, 2013, pp. 1-6. doi: 10.11648/j.epes.20130201.11

\begin{abstract}
The primary purpose in constructing equivalents is to represent a portion of a network containing many buses but having only a few "boundary buses" by a reduced network containing only the boundary buses and, perhaps, a few selected buses from within the original sub-network. The equivalent constructed gives an exact reproduction of the self and transfer impedances of the external system as seen from its boundary buses. PowerFactory's network reduction algorithm produces an equivalent representation of the reduced part of the network and calculates its parameters. This equivalent representation is valid for both load flow and short-circuit calculations, including asymmetrical faults (that is, single-phase faults).
\end{abstract}

Keywords: Boundary Buses, Reduced Network, Powerfactory, Load Flow, Short-Circuit Calculation

\section{Introduction}

A typical application of network reduction is a project where a specific network has to be analyzed, but this network cannot be studied independent of a neighboring network of the same or a higher/lower voltage level. In such cases, one option is to model both networks in detail for calculation purposes. There may, however, be situations when it is not desirable to perform studies with the complete model. Such situations may include, for example, cases when the calculation times would increase significantly or when the data of the neighboring network is confidential and cannot be published in detail. In these cases it is common practice to provide a representation of the neighboring network, which contains the interface nodes (connection points) that may be connected by equivalent impedances and voltage sources [1].

Network reduction for load flow is an algorithm based on sensitivity matrices. The basic idea is that the sensitivities of the equivalent grid, measured at the connection points in the retained grid, must be equal to the sensitivities of the grid that has been reduced. This means that for a given (virtual) set of $\Delta \mathrm{P}$ and $\Delta \mathrm{Q}$ (active an reactive power variations) injections in the branches, from the retained grid to the grid to be reduced, the resulting $\Delta \mathrm{u}$ and $\Delta \phi$ (voltage magnitude and voltage phase angle variations) in the boundary nodes must be the same for the equivalent grid, as those that would have been obtained for the original grid (within a user defined tolerance).

Network reduction for short-circuit is an algorithm based on nodal impedance/nodal admittance matrices. The basic idea is that the impedance matrix of the equivalent grid, measured at the connection points in the retained grid, must be equal to the impedance matrix of the grid to be reduced (for the rows and columns that correspond to the boundary nodes). This means that for a given (virtual) additional $\Delta \mathrm{I}$ injection (variation of current phasor) in the boundary branches, from the retained grid to the grid to be reduced, the resulting $\Delta \mathrm{u}$ in the boundary nodes must be the same for the equivalent grid, as those that would have been obtained for the original grid (within a user defined tolerance). This must be valid for positive sequence, negative sequence, and zero sequence cases, if these are to be considered in the calculation (unbalanced short-circuit equivalent) [2-7].

\section{Methodology}

Power system network matrices [8]:

$$
I_{b u s}=Y_{b u s} V_{b u s}
$$

where 
$I_{b u s}$ is the vector of the injected bus currents;

$V_{b u s}$ is the vector of bus voltages measured from the reference node.

$Y_{b u s}$ is known as the bus admittance matrix.

In a power system, the current injection is always zero at buses where there are no external loads or generators connected; such nodes may be eliminated. Then for an n-bus system, the reduced admittance matrix by eliminating node $\mathrm{k}$ is:

$$
I_{k}=0
$$

where node $\mathrm{k}$ has zero current injection, therefore,

$$
I_{k}=0
$$

The new bus admittance matrix is:

$$
Y_{i j}^{(\text {new })}=Y_{i j}-\frac{Y_{i j} Y_{k j}}{Y_{k k}}
$$

where $\mathrm{i}, \mathrm{j}=1,2, \ldots$, n. $i, j \neq k$

In stability studies, all nodes are eliminated except for the internal generator nodes and obtain the Y matrix for the reduced network:

$$
\mathrm{I}=\mathrm{YV}
$$

where

$$
I=\left[\begin{array}{c}
I_{n} \\
0
\end{array}\right]=\left[\begin{array}{ll}
Y_{n n} & Y_{n r} \\
Y_{r n} & Y_{r r}
\end{array}\right]\left[\begin{array}{c}
V_{n} \\
V_{r}
\end{array}\right]
$$

The matrix in equation (5) is the reduced matrix Y. It has dimensions ( $\mathrm{n} \times \mathrm{n})$, where $\mathrm{n}$ is the number of generators.

Network reduction can be applied only to those nodes that have zero injection current. An equivalent represents a network, which contains many buses but only a few boundary buses, by a reduced network that contains only the boundary buses and a few of the original buses. Equivalents are used in two circumstances: both to allow larger areas of major interconnected systems to be represented in studies and also to achieve improved computational speed in simulations by removing buses and branches that influence system behavior.

A boundary cuts a set of tie-lines between areas or otherwise identifiable sections of a network but passes through no buses. A boundary bus is part of only one area. An equivalent makes more efficient use of storage when the ratio of branches to buses in the equivalent is reduced. As a general rule, however, reducing a system into a number of small equivalents is more efficient than reducing of a large system in one step to produce a single equivalent [9-11].

\section{Network Modeling in PowerFactory}

Figure 1 shows IEEE nine-bus system with three synchronous generators. Generator $\left(G_{1}\right)$ was selected the swing generator. The transformer, load and generators data are given in Table 1, 2 and 3 respectively. The network model contains the electrical and graphical information for the grid. To further enhance manageability, this information is split into two subfolders: diagrams and network data. An additional subfolder, Variations, contains all expansion stages for planning purposes. The network model folder contains the all graphical and electrical data which defines the networks and the single line diagrams of the power system under study. This set of data is referred as the network data model.

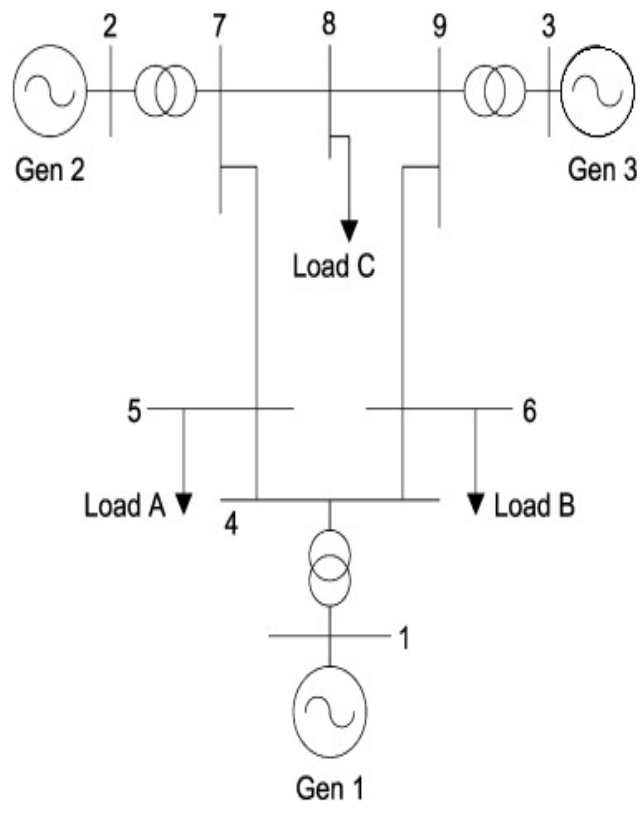

Figure 1. IEEE three-machine, nine-bus test system [12].

Table 1. Transformers data.

\begin{tabular}{lccc}
\hline & $\mathbf{T}_{\mathbf{r} 1}$ & $\mathbf{T}_{\mathbf{r} 2}$ & $\mathbf{T}_{\mathbf{r} 3}$ \\
\hline MVA & 250 & 200 & 150 \\
HV (kV) & 230 & 230 & 230 \\
LV (Kv) & 16.5 & 18 & 13.8 \\
\hline
\end{tabular}

Table 2. Loads data.

\begin{tabular}{llll}
\hline & $\operatorname{Load}_{\mathbf{A}}$ & $\operatorname{Load}_{\mathbf{B}}$ & $\operatorname{Load}_{\mathbf{C}}$ \\
\hline $\mathrm{MV}$ & 125 & 90 & 100 \\
$\mathrm{Mvar}$ & 50 & 30 & 35 \\
\hline
\end{tabular}


Table 3. Generators data.

\begin{tabular}{|c|c|c|c|}
\hline Parameters & $\mathrm{G}_{1}$ (swing) & $\mathbf{G}_{2}$ & $\mathbf{G}_{3}$ \\
\hline Apparent power (MVA) & 247.5 & 192 & 128 \\
\hline Voltage $(\mathrm{kV})$ & 16.5 & 18 & 13.8 \\
\hline Power factor & 1 & 0.85 & 0.85 \\
\hline Active power (MW) & - & 150 & 85 \\
\hline Reactive power (Mvar) & - & 6.7 & -10.9 \\
\hline Voltage (p.u.) & 1.04 & 1.025 & 1.025 \\
\hline Rotor type & salient & round & round \\
\hline Syn. reactances, $x_{d}$ & 0.36 & 1.72 & 1.68 \\
\hline Syn. reactances, $\mathrm{x}_{\mathrm{q}}$ & 0.24 & 1.66 & 1.61 \\
\hline Sub. transient $\left(x_{d}^{\prime \prime}\right)$ & 0.2 & 0.2 & 0.2 \\
\hline Sub. transient $\left(x_{a}^{\prime \prime}\right)$ & 0.1 & 0.12 & 0.2 \\
\hline $\operatorname{transient}\left(x_{d}^{\prime}\right)$ & 0.15 & 0.23 & 0.23 \\
\hline transient $\left(x_{a}^{\prime}\right)$ & 0.00 & 0.378 & 0.32 \\
\hline Rotor resistances & 0.00 & 0.005 & 0.00 \\
\hline Time constant $T_{d}^{\prime}$ & 3.73 & 0.8 & 0.806 \\
\hline Time constant $T_{d}^{\prime \prime}$ & 0.05 & 0.05 & 0.05 \\
\hline Accel. Time constant & 9.55 & 4.162 & 2.765 \\
\hline Leakage reactances $x$ & 0.083 & 0.141 & 0.0949 \\
\hline
\end{tabular}

\section{Network Reduction in Power Factory}

The Network Reduction function, by default, retains the original network data. The boundary, necessary to split the grid into the part to be reduced, was defined and the part to retain its detailed representation. To ensure that the boundary splits the network into two regions, Check Split button in the ElmBoundary dialogue could be used.
The distribution network in Fig. 1 is fed by two busbars, 'Bus 5' and 'Bus 6', in the center of the transmission system. It is represented by Load A and Load B and the corresponding two transformers. If the distribution system is to be studied in details, while the transmission system is to be represented only by its equivalent model. The transmission system has a connection to another transmission system, which is represented by the External Grid and the connecting line. This second transmission system shall remain as it is, the aim of the paper is to reduce only one transmission system. The "Boundary" (shown by dotted line in Figure 1) defines which part shall be reduced [1]. After this, the network is reduced using PowerFactory. The network reduction procedure will automatically create a new variation/system stage to represent the original grid.

In the new system stage(s), the part of the grid which was not reduced retains its full representation, whereas the part that was reduced is erased and the new simplified grid representation is added, with the connections to the other part of the grid (that is, to the part which is not reduced). The new system stage(s) will therefore represent the combined grid (retained grid and reduced equivalent). The new system stages will be automatically activated in the active study case. To calculate short-circuit equivalent, this option is used to specify whether the short-circuit equivalent shall be calculated (option enabled) or not (option disabled). PowerFactory currently supports only the complete shortcircuit calculation method [1]. Figure 2 shows elements in the defined boundary. These are to be reduced to an equivalent three AC voltage sources (ElmVac) and three common impedances (ElmZpu) connecting the remaining busbars.

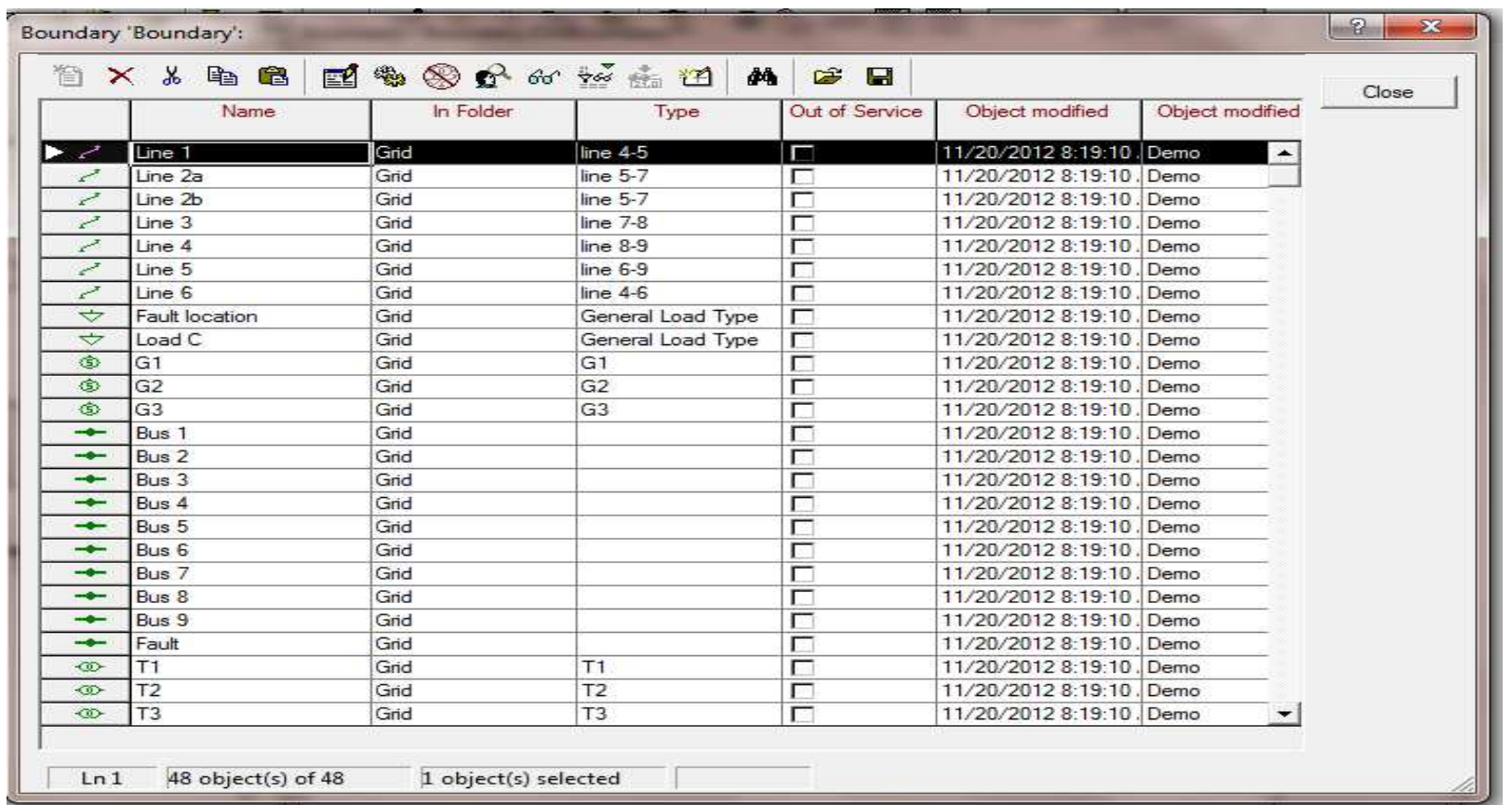

Figure 2. Elements in the defined boundary. 


\section{Results and Discussion}

The network reduction was carried out with PowerFactory by DIgSILENT version 14.1 [1]. The equivalent model for power injection is extended ward equivalent method.
The short-circuit method used is complete method with 0.1 seconds as break time, while the fault clearing time is 1 second.

Figures 3 and 4 show PowerFactory command dialogue for $\mathrm{AC}$ voltage source and common impedance respectively.

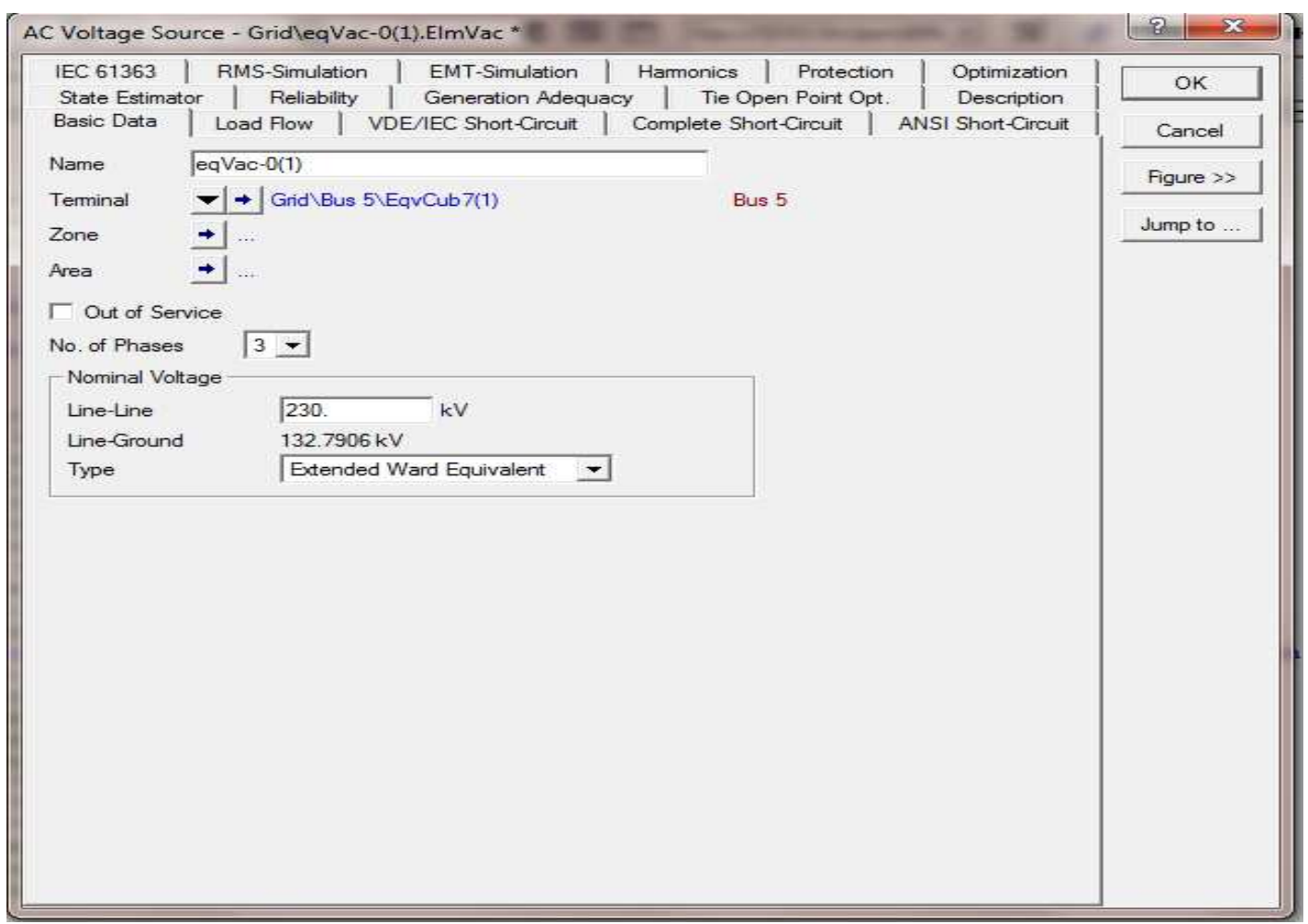

Figure 3. Command dialogue for AC voltage source.

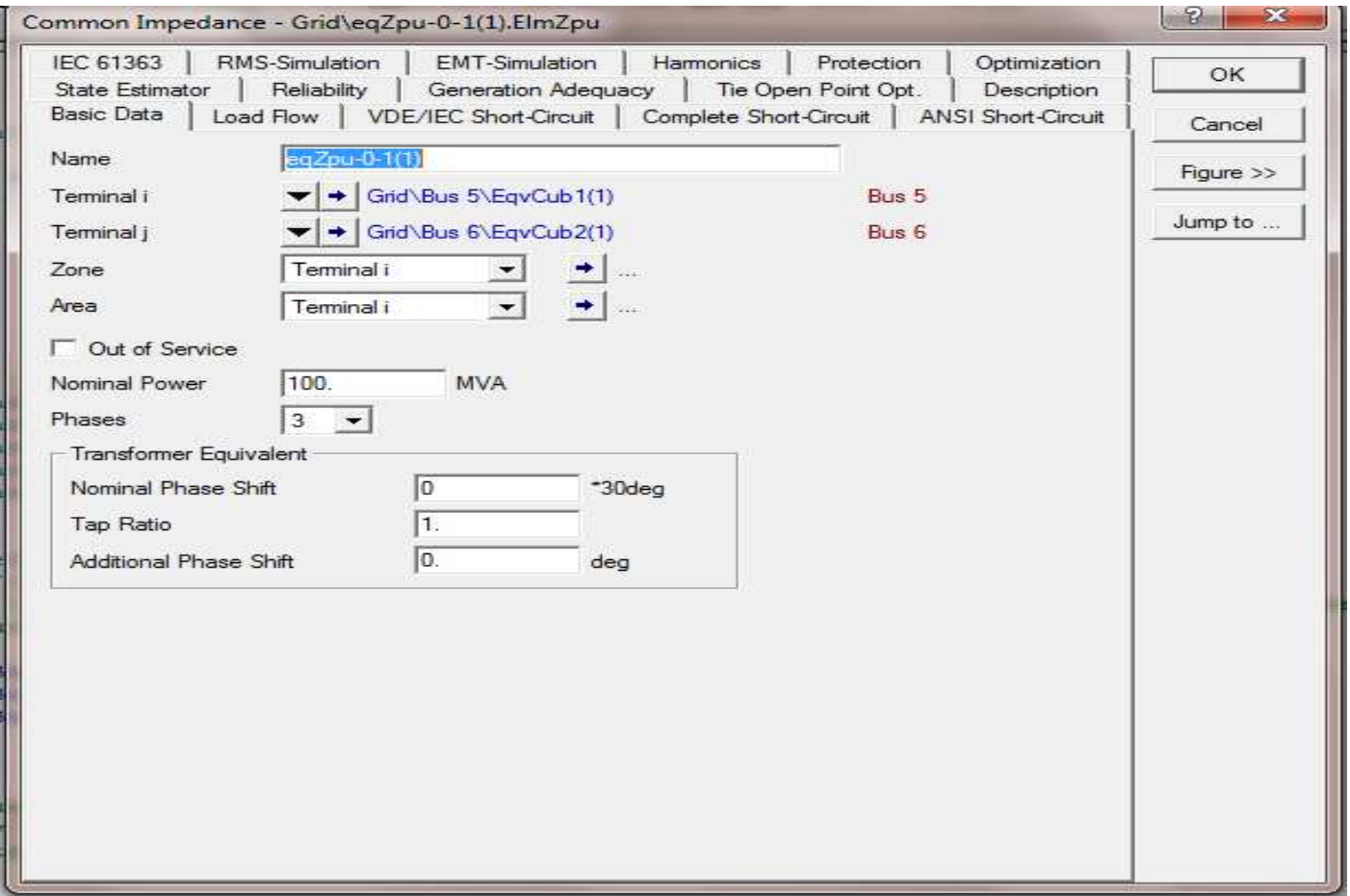

Figure 4. Command dialogue for common impedance. 
The transmission network was reduced to an equivalent representation by three AC voltage sources (eqVac-0 at bus 6; eqVac-1 at bus 5 and eqVac-2 at bus 4) and three common impedances (eqZpu-0-1 between buses 6 and 5; eqZpu-0-2 between buses 6 and 4; eqZpu-1-2 between buses 5 and 4 ) connecting the remaining busbars. The resulting load flow and method short-circuit parameters are given in Table $4-6$.

Table 4. Parameters of the AC voltage sources.

\begin{tabular}{llll}
\hline Parameters & eqVac-0 & eqVac-1 & eqVac-2 \\
\hline Terminal voltage (kV) & 230 & 230 & 230 \\
Active power (MW) & 28.211 & 78.667 & -0.00068 \\
Reactive power (Mvar) & 27.89 & 21.389 & 17.6732 \\
$\mathrm{~V}_{\text {mag }}(\mathrm{p} . \mathrm{u})$. & 1.0143 & 1.0026 & 1.02871 \\
$\mathrm{r}_{\text {ward }}(\mathrm{ohm})$ & 0.00 & 0.00 & 0.00 \\
$\mathrm{X}_{\text {ward }}(\mathrm{ohm})$ & 312.85 & 114.68 & -1583.6 \\
$\mathrm{r}_{\text {short }}(\mathrm{ohm})$ & 48.318 & 13.530 & -13.298 \\
$\mathrm{X}_{\text {short }}(\mathrm{ohm})$ & 247.67 & 132.06 & -13171.2 \\
\hline
\end{tabular}

Table 5. Parameters of the common impedances (load-flow).

\begin{tabular}{lllll}
\hline Components & \multicolumn{2}{l}{ Load flow } & & \\
\hline & positive sequence & negative & zero \\
\hline & (real) & (imag.) & (re, im) & (re, im) \\
eqZpu-0-1 & 0.1151325 & 0.4091812 & 0 & 0 \\
eqZpu-0-2 & 0.0170001 & 0.0920005 & 0 & 0 \\
eqZpu-1-2 & 0.01 & 0.0850005 & 0 & 0 \\
\hline
\end{tabular}

Table 6. Parameters of the common impedances (complete short-circuit).

\begin{tabular}{lllll}
\hline Components & \multicolumn{2}{l}{ complete short-circuit } & & \\
\hline & positive sequence & negative & zero \\
\hline & (real) & (imag.) & (re, im.) & (re, im) \\
eqZpu-0-1 & 0.150755 & 0.150755 & 0 & 0 \\
eqZpu-0-2 & 0.0170326 & 0.0919994 & 0 & 0 \\
eqZpu-1-2 & 0.0100325 & 0.0850005 & 0 & 0 \\
\hline
\end{tabular}

The load flow calculation in the reduced network gives the same results for the distribution network as for the original (non-reduced) network as shown in Table 7 and 8 . In Table 7, the buses 1, 4, 5 and 6 busbars report for the reduced network is the same as the busbars report for buses 1 , 4, 5 and 6 for non-reduced network as shown in Table 8 .
Table 7. Load flow (busbars) report for non-reduced network.

\begin{tabular}{lllll}
\hline Busbars & rated Voltage & \multicolumn{2}{l}{ Bus - Voltage } & deg. \\
\hline & $\mathbf{( k V )}$ & $\mathbf{( k V )}$ & $\mathbf{( p . u . )}$ & \\
\hline Bus 1 & 16.5 & 17.16 & 1.04 & 0 \\
Bus 2 & 18 & 18.45 & 1.025 & 4.81 \\
Bus 8 & 230 & 232.6 & 1.011 & 147.17 \\
Bus 9 & 230 & 237.14 & 1.031 & 149.23 \\
Bus 3 & 13.8 & 14.14 & 1.025 & 1.93 \\
Bus 6 & 230 & 233.29 & 1.014 & 144.99 \\
Bus 4 & 230 & 236.61 & 1.029 & 147.31 \\
Bus 5 & 230 & 230.6 & 1.003 & 145.6 \\
Bus 7 & 230 & 234.51 & 1.02 & 149.66 \\
\hline
\end{tabular}

Table 8. oad flow (busbars) report for reduced network.

\begin{tabular}{lllll}
\hline Busbars & rated Voltage & \multicolumn{2}{l}{ Bus - Voltage } & deg. \\
\hline & $\mathbf{( k V )}$ & $\mathbf{( k V )}$ & $\mathbf{( p . u . )}$ & \\
\hline Bus 1 & 16.5 & 17.16 & 1.04 & 0 \\
Bus 6 & 230 & 233.29 & 1.014 & 144.99 \\
Bus 4 & 230 & 236.61 & 1.029 & 147.31 \\
Bus 5 & 230 & 230.6 & 1.003 & 145.6 \\
\hline
\end{tabular}

\section{Conclusion}

PowerFactory's network reduction algorithm produces an equivalent representation of the reduced part of the network and calculates its parameters. This equivalent representation is valid for both load flow and short-circuits calculations, including asymmetrical faults (that is, single-phase faults). By using small, equivalent networks, the computational requirements can be significantly reduced. Network reduction is usually performed by computing impedances and by eliminating unnecessary elements.

This reduction usually results in a highly dense impedance matrix; therefore, using the reduced network may not significantly increase efficiency. Equivalent networks have been used for load flow and short circuit studies because they can reproduce the same voltages and currents of the remaining buses as the original systems do [13]. A load flow calculation or a short-circuit calculation in the reduced network gives the same results for the distribution network as for the original (non-reduced) network.

\section{Nomenclature}

p.u.: per unit;

$\mathrm{HV}$ : high voltage;

LV: low voltage; 
MW: megawatts;

Mvar: mega volt ampere reactive;

$\mathrm{kV}$ : kilo voltage;

$\mathrm{V}_{\text {vag }}$ : voltage magnitude for extended ward method;

$\mathrm{R}_{\mathrm{ward}}$ : resistance for the extended ward;

$\mathrm{X}_{\text {ward }}$ : reactance for the extended ward;

$\mathrm{r}_{\text {short }}$ : positive-sequence complete short-circuit resistance;

$\mathrm{X}_{\text {short }}$ : positive-sequence complete short-circuit reactance;

\section{References}

[1] DIgSILENT PowerFactory Version 14.1 Tutorial, DIgSILENT GmbH Heinrich-Hertz-StraBe 9, 72810 Gomaringen, Germany, May, 2011.

[2] Buygi M. O., H. M. Shanechi, G. Balzer, and M. Shahidehpour, Transmission Planning Approaches in Restructured Power Systems, IEEE Powertech, June 2006.

[3] Ward J. B.. Equivalent circuits for power flow studies. AIEE Trans. Power App. Syst., 8:373-380, February 1949.

[4] Singh H.K. and Srivastava S.C.. A sensitivity based network reduction technique for power transfer assessment in deregulated electricity environment. Transmission and Distribution Conference and Exhibition: Asia Pacific, IEEE/PES, October 2002.
[5] Tinney W. F. and J. M. Bright, "Adaptive reductions for power flow equivalents," IEEE Trans. Power Syst., vol. 6, no. 2, pp. 613-621, May 1991.

[6] Cheng X. and T. J. Overbye, "PTDF-based power system equivalents," IEEE Trans. Power Syst., vol. 20, no. 4, pp. 1868-1876, Nov. 2005.

[7] Enns M. K. and J. J. Quada, "Sparsity-enhanced network reduction forfault studies," IEEE Trans. Power Syst., vol. 6, no. 2, pp. 613-621, May 1991.

[8] Saadat, H. 'Power System Analysis'. McGraw- Hill International Editions, 1999.

[9] P.M. Anderson \& A.A. Fouad, 'Power System Control and Stability', 2nd edition, IEEE Press Power Engineering Series, Wiley-Interscience, 2003.

[10] Arthur R. Bergen \& Vijay Vittal, 'Power System Analysis', 2nd edition, Prentice Hall, Inc., 2000.

[11] Yao-nan Yu, 'Electric Power System Dynamics', Academic Press, Inc., 1983.

[12] www.sciencedirect.com.

[13] Hyungseon Oh, 'A New Network Reduction Methodology for Power System Planning Studies', IEEE Transactions on Power Systems, Vol. 25, No. 2, May 2010. 